\title{
Distinct subcellular localization of tRNA-derived fragments in the infective metacyclic forms of Trypanosoma cruzi
}

\author{
Larissa Reifur ${ }^{1,3}$, Maria Rosa Garcia-Silva ${ }^{2}$, Saloê Bispo Poubel' ${ }^{1}$, Lysangela Ronalte Alves ${ }^{1}$, \\ Paulo Arauco', Diane Kelly Buiar ${ }^{3}$, Samuel Goldenberg1', Alfonso Cayota ${ }^{2}$, Bruno Dallagiovanna ${ }^{1 /+}$

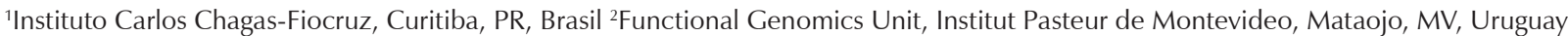 \\ ${ }^{3}$ Departamento de Patologia Básica, Setor de Ciências Biológicas, Universidade Federal do Paraná, Curitiba, PR, Brasil
}

Small non-coding RNAs derived from transfer RNAs have been identified as a broadly conserved prokaryotic and eukaryotic response to stress. Their presence coincides with changes in developmental state associated with gene expression regulation. In the epimastigote form of Trypanosoma cruzi, $t R N A$ fragments localize to posterior cytoplasmic granules. In the infective metacyclic form of the parasite, we found tRNA-derived fragments to be abundant and evenly distributed within the cytoplasm. The fragments were not associated with polysomes, suggesting that the tRNA-derived fragments may not be directly involved in translation control in metacyclics.

Key words: Trypanosoma cruzi - tRNA-derived fragments - subcellular localisation

The non-infective epimastigote form of Trypanosoma cruzi undergoes metabolic and morphological adaptations to differentiate into the pathogenic metacyclic trypomastigote form, which causes Chagas disease in mammals (Figueiredo et al. 2000). Morphological changes are associated with and preceded by a shift in metabolism, which is correlated with the differential expression of several genes (Minning et al. 2009). Although the metabolism of the replicative epimastigote is fully active, with constitutive polycistronic transcription, starvation reduces the transcriptional rates of the RNA polymerases in metacyclics (Ferreira et al. 2008). Despite the reduced level of transcription, translation is detected in these non-replicative forms, indicating that gene expression control in metacyclics occurs primarily at the post-transcriptional level (reviewed in Haile \& Papadopoulou 2007).

Considering the almost exclusive post-transcriptional control of gene expression in T. cruzi, it would be surprising if this organism did not have an alternative pathway to compensate for the absence of an RNA interference system. A myriad of small non-coding RNAs have been reported in trypanosomatids (Dumas et al. 2006, Garcia-Silva et al. 2010, Michaeli et al. 2012), canonical microRNAs, and siRNAs have not been detected in T. cruzi (Franzén et al. 2011). Short (20-35 nt) RNAs derived from tRNAs were first observed in cytoplasmic granules in the epimastigote form of $T$. cruzi after an ini-

Financial support: FIOCRUZ, Araucaria Foundation (18.456) (to LR) SG, LRA and BD received fellowships from CNPq, SBP received a fellowship from CAPES and DKB received a fellowship from UFPR/TN. + Corresponding author: brunod@tecpar.br

Received 20 December 2011

Accepted 10 May 2012 tial fingerprint sequencing of 348 clones (Garcia-Silva et al. 2010). Although $26 \%$ of the sequenced clones represented tRNA-derived fragments, a more thorough sequencing of the small RNAs from epimastigotes showed that $65.3 \%$ of more than 282.000 clones represented tRNA-derived fragments (Franzén et al. 2011). The composition of the small RNA population in T. cruzi was strikingly different from that observed in Trypanosoma brucei, reflecting clear differences in the molecular biology of the two parasites (Michaeli et al. 2012). Nonetheless, the relative abundance of tRNA-derived fragments could be related to gene expression control under various types of cellular stress, as has been suggested for prokaryotes, yeast, mammalian cells, the protozoans Giardia lamblia and Tetrahymena thermophila (Lee \& Collins 2005, Li et al. 2008, Pederson 2010).

In this study, we conducted further analyses of the relative abundance and subcellular localization of T. cru$z i$ tRNA-derived fragments during the infective stage of the parasite. We used T. cruzi metacyclic trypomastigotes derived from Dm28c epimastigotes cultured in vitro, as described by Contreras et al. (1985). Total RNA was extracted using TRIzol (Invitrogen) and size fractionated on a denaturing $15 \%$ polyacrylamide gel electrophoresis (PAGE) gel. Subsequently, 18-40 nt RNAs were excised from the gel, purified and cloned as described by GarciaSilva et al. (2010). To recover the small RNAs, specific oligonucleotide adaptors containing Ban 1 restriction sites were ligated to the 5' and ' ' ends. The RNAs were then reverse transcribed, amplified by polymerase chain reaction for 10 cycles, Ban 1-digested, concatamerized, cloned into the pGEM T-easy vector (Promega Corp) and sequenced. Analyses were performed using the public GenBank (ncbi.nlm.nih.gov/genbank), GeneDB (genedb. org) and TriTrypDB (tritrypdb.org/tritrypdb) databases.

From a total of 844 clones analysed, 509 sequences aligned with the T. cruzi genome, whereas $2.16 \%$ showed no matches, indicating that these sequences may correspond to regions of the genome that have not been se- 
quenced, exogenous contaminating DNA or differences between the reference strain and the strain used in this study. No sequences matched small nuclear RNA sequences, but $0.98 \%$ mapped to small nucleolar RNAs, $2.95 \%$ mapped to intergenic regions, 6.09\% mapped to mRNAs and $24.56 \%$ mapped to rRNAs. Most of the cloned sequences $(63.26 \%)$ were fragments derived from tRNAs that appeared to be the result of a specific cleavage at or around the anticodon loop (Supplementary data). The tRNA fragments averaged $33 \mathrm{nt}$ in length and were mostly derived from the $3^{\prime}$ end $(86.96 \%)$ of a restricted group of isoacceptors. In contrast with the RNAs obtained from an epimastigote population sequenced by Garcia-Silva et al. (2010), the tRNA-derived fragments obtained in this study from metacyclics exhibited differences with respect to abundance (tRNA fragments composed $63.26 \%$ of the small RNAs in metacyclics versus $26 \%$ in epimastigotes), orientation (most of the tRNA fragments were derived from the 3' side of the tRNAs in metacyclics vs. the 5' side in epimastigotes) and origin (in metacyclics, fragments were derived mostly from tRNA ${ }^{\text {Glu }}$, tRNA ${ }^{\text {Thr }}$ and tRNA ${ }^{\text {Vall }}$; in epimastigotes, they were generally derived from tRNA ${ }^{\text {Asp }}$ and tRNA ${ }^{\mathrm{Glu}}$ ). The relative abundances of most fragments did not correspond to either tRNA gene copy number or codon usage (Horn 2008, Padilla-Mejia et al. 2009), consistent with previous reports (Franzén et al. 2011). As reported by Franzén et al. (2011), we observed that the majority of the fragments were derived from the 3' side of the tRNAs and a significant proportion of these fragments (24.53\%) carried the 3' CCA sequence, indicating that both mature and pre-tRNAs undergo the cleavage process. The biological importance of these tRNA fragments is unknown, but we expected that a higher percentage of these fragments would be observed in metacyclics because stressed epimastigotes showed only a slight increase in tRNA-derived fragments (Garcia-Silva et al. 2010). This phenomenon is likely observed because tRNA cleavage is a conserved process in cells under various types of stress and metacyclic trypomastigotes are the product of epimastigote differentiation triggered by nutritional stress (Contreras et al. 1985). A comparison of our results with those of Garcia-Silva et al. (2010) demonstrates that the percentage of tRNA fragments is clearly higher in metacyclics. In contrast, this difference is not observed by Franzén et al. (2011). Moreover, the epimastigote tRNA-derived fragments sequenced by Franzén et al. (2011) are mostly derived from the 3' arm of tRNA ${ }^{\text {His }}$. These discrepancies could be due to differences in the strains analysed in the two studies and to the cloning and sequencing methods used. Franzén et al. (2011) used a different strain (CL Brener) and analysed a much larger number of clones using RNAseq, which provided a higher coverage of the parasite genome.

The cloned RNA sequences were aligned using the LocARNA server (Will et al. 2007) and the secondary structures identified using the RNAalifold server (Bernhart et al. 2008) from the Vienna RNA package and were adjusted manually according to the secondary structural domains of canonical tRNAs. The predicted secondary structures adopted by the most abundant 3' tRNA frag- ments revealed that, upon cleavage around the anticodon

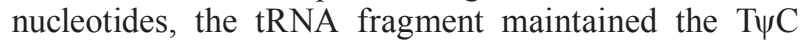
loop conformation and was extended a few extra base pairs into the double-stranded stem, resulting in an energetically favourable structure (Supplementary data). Although this result is based only on computational calculations and does not confirm the true molecular structure in vivo, the fact that the most abundant tRNA-derived fragments can assume a similar, relatively stable configuration suggests a structural (or functional) significance.

The subcellular localization of the tRNA-derived fragments in the metacyclic forms was evaluated through fluorescence in situ hybridisation (FISH) using probes complementary to the 5' and 3' ends of tRNA Glu-Uuc (the most abundantly cloned tRNA fragment). FISH assays showed that these fragments are dispersed throughout the cytoplasm in metacyclics, whereas stressed epimastigotes exhibited the same posterior granular distribution as non-stressed epimastigotes (Fig. 1). To further analyze the cellular localization of the tRNA-derived fragments, metacyclic cells were gently lysed in lysis buffer $(300 \mathrm{mM} \mathrm{KCl}, 10 \mathrm{mM} \mathrm{MgCl}, 10 \mathrm{mM}$ Tris-HCl $7.4 \mathrm{pH}$ and $0.5 \% \mathrm{NP} 40$ ) for $5 \mathrm{~min}$. Two fractions were collected: the supernatant, containing only the soluble cytosol contents (S), and the insoluble fraction, or pellet $(\mathrm{P})$, containing insoluble organelles, vesicles and cellular membranes. Both fractions were loaded on a sodium dodecyl sulphate-PAGE $(10 \%)$ and transferred onto Hybond-C membranes (Amersham). Western blots were performed using antibodies to TcPUF6 (1/250), a cytosolic RNA binding protein (Dallagiovanna et al. 2005), and dynamin (1/200), a membrane protein present in endocytic vesicles (Pucadyil \& Schmid 2009) (Fig. 2F, G). Northern blots were conducted using total RNA extracted from the two cell fractions $(\mathrm{S}$ and $\mathrm{P})$ and a ra-
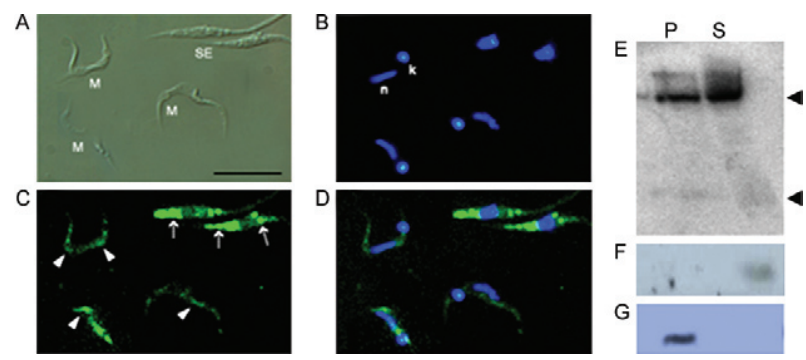

Fig. 1: subcellular localization of tRNA-derived RNAs in metacyclic trypomastigote forms. A: differential imaging contrast of metacyclic (M) and stressed epimastigote forms (SE); B: 4'-6-diamidino-2-phenylindole staining was used as a reference to identify nuclei (n) and kinetoplast (k); C: fluorescein isothiocyanate (FITC)-labeled probes recognizing the 3' fragment tRNA Glu-UUC (5'TTCCGATACCGGGAATCGA3') in SE (arrows) and M forms (arrowheads); D: merged images $(\mathrm{Bar}=10 \mu \mathrm{m})$. Cells were observed under an Olympus IX 81 microscope coupled to a Hamamatsu Orca-ER camera (Diagnostic Instruments); E: total RNA from soluble (S) and insoluble $(\mathrm{P})$ metacyclic cell fractions were analyzed with the radioactively labelled probe 3 'tRNA ${ }^{\text {Glu-UUC }}$, as in Garcia-Silva et al. (2010). Western blot assays with F (anti TcPUF6) and G (anti-Dynamin) sera were performed to assess the identity of both fractions. 

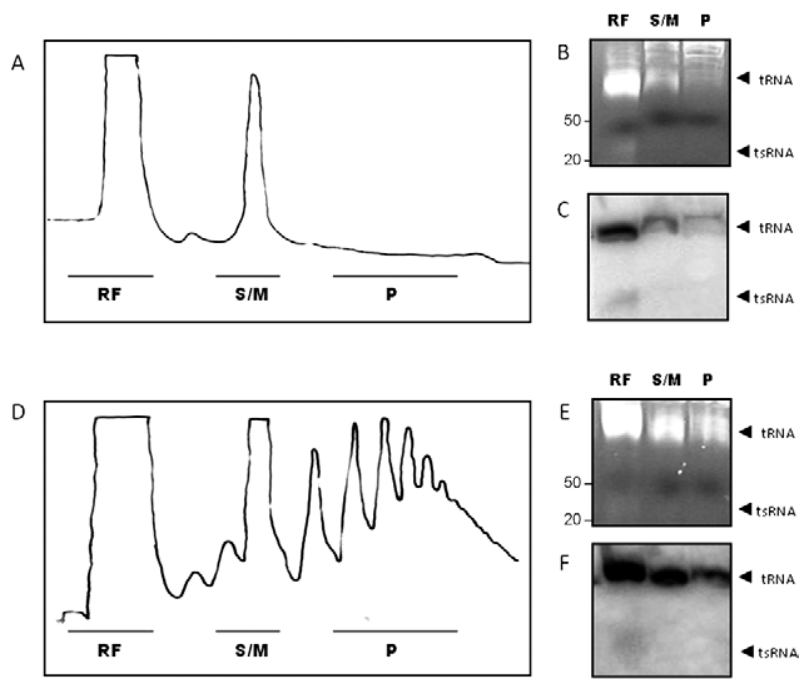

Fig. 2: tRNA-derived fragments are not associated with polysomes. Polysome profiles of (A) metacyclic trypomastigote and (D) epimastigote forms. Fractions were collected and pooled (RF: ribosome free; S/M: subunits and monomers; P: polysomes). RNA was purified and separated into $15 \%$ acrylamide gels (B, E). Northern blots of metacyclic (C) and epimastigote RNA (F) analyzed with the radioactively labelled probe tRNA ${ }^{\text {Glu-UUC }}$, as in Fig. 1.

diolabeled probe specific to the tRNA ${ }^{\text {Glu-UUC }}$ fragment. The tRNA-derived fragments were detected in both fractions, supporting the dispersed pattern observed in the immunofluorescence assays (Fig. 2E). The signal observed for the insoluble cellular fraction could indicate the presence of these RNAs in smaller intracellular vesicles. Nonetheless, their dispersed pattern in the cytoplasm in metacyclics contrasts with the granular and posterior localization in epimastigotes, in which a partial co-localization with reservosomes has been inferred. The dismantling of the tRNA fragments in metacyclics could be correlated with the absence of reservosomes in this life stage (Figueiredo et al. 2000) and is consistent with the described cytoplasmic localization of the type II tRNA-derived fragments. Type II tRNA-derived fragments are most likely generated in the cytosol by RNaseZ cleavage and by RNA polymerase III termination and these fragments have been found to coimmunoprecipitate with Argonaute proteins (Elbarbary et al. 2009, Haussecker et al. 2010). This dramatic change in subcellular localization is puzzling and suggests distinct roles for these molecules during the different stages of the parasite life cycle.

To determine whether the dispersed tRNA-derived fragments were associated with translating polysomes, metacyclic and epimastigote forms of T. cruzi were treated with cycloheximide and the polysomes were purified and separated on sucrose gradients, as previously described (Nardelli et al. 2007) (Fig. 2A, D). Parasite extracts were also treated with puromycin as a negative control (not shown). The polysomes, monosomes and ribosome-free fractions were pooled and the total RNA was extracted. After acrylamide gel separation and staining, a high concentration of small RNAs was observed in the ribosome-free pool for the metacyclic and epimastigote forms and a faint small RNA signal was observed in the ribosome-containing fractions (Fig. 2B, E). Northern blot analysis confirmed that tRNAderived fragments from metacyclics and epimastigotes were concentrated in the ribosome-free fractions (Fig. $2 \mathrm{C}, \mathrm{F}$ ). Therefore, tRNA-derived fragments may not be related to the repression of the translational machinery. The knockdown of the tRNA-derived fragments and structural analysis should be used to help uncover the biological importance of these RNAs.

\section{ACKNOWLEDGEMENTS}

To Dr Donna J Koslowsky, Dr Laura E Yu and the reviewers, for critical reading of the manuscript, to Stenio Fragoso and Rosana Gonçalves, for the anti-Dynamin serum, and Nilson Fidencio, for technical assistance.

\section{REFERENCES}

Bernhart SH, Hofacker IL, Will S, Gruber AR, Stadler PF 2008. RNAalifold: improved consensus structure prediction for RNA alignments. BMC Bioinformatics 9: 474.

Contreras VT, Salles JM, Thomas N, Morel CM, Goldenberg S 1985. In vitro differentiation of Trypanosoma cruzi under chemically defined conditions. Mol Biochem Parasitol 16: 315-327.

Dallagiovanna B, Perez L, Sotelo-Silveira J, Smircich P, Duhagon MA, Garat B 2005. Trypanosoma cruzi: molecular characterization of TcPUF6, a Pumilio protein. Exp Parasitol 109: 260-264.

Dumas C, Chow C, Muller M, Papadopoulou B 2006. A novel class of developmentally regulated noncoding RNAs in Leishmania. Eukaryot Cell 5: 2033-2046.

Elbarbary RA, Takaku H, Uchiumi N, Tamiya H, Abe M, Takahashi M, Nishida H, Nashimoto M 2009. Modulation of gene expression by human cytosolic tRNase Z(L) through 5'-half-tRNA. PLOS ONE 4: e5908.

Ferreira LR, Dossin F de M, Ramos TC, Freymuller E, Schenkman S 2008. Active transcription and ultrastructural changes during Trypanosoma cruzi metacyclogenesis. An Acad Bras Cienc 80: 157-166.

Figueiredo RC, Rosa DS, Soares MJ 2000. Differentiation of Trypanosoma cruzi epimastigotes: metacyclogenesis and adhesion to substrate are triggered by nutritional stress. J Parasitol 86: $1213-1218$.

Franzén O, Arner E, Ferella M, Nilsson D, Respuela P, Carninci P, Hayashizaki Y, Aslund L, Andersson B, Daub CO 2011. The short non-coding transcriptome of the protozoan parasite Trypanosoma cruzi. PLoS Negl Trop Dis 5: e1283.

Garcia-Silva MR, Frugier M, Tosar JP, Correa-Dominguez A, Ronalte-Alves L, Parodi-Talice A, Rovira C, Robello C, Goldenberg S, Cayota A 2010. A population of tRNA-derived small RNAs is actively produced in Trypanosoma cruzi and recruited to specific cytoplasmic granules. Mol Biochem Parasitol 171: 64-73.

Haile S, Papadopoulou B 2007. Developmental regulation of gene expression in trypanosomatid parasitic protozoa. Curr Opin Microbiol 10: 569-577.

Haussecker D, Huang Y, Lau A, Parameswaran P, Fire AZ, Kay MA, 2010. Human tRNA-derived small RNAs in the global regulation of RNA silencing. RNA 16: 673-695.

Horn D 2008. Codon usage suggests that translational selection has a major impact on protein expression in trypanosomatids. $B M C$ Genomics 9: 2. 
Lee SR, Collins K 2005. Starvation-induced cleavage of the tRNA anticodon loop in Tetrahymena thermophila. J Biol Chem 280: 42744-42749.

Li Y, Luo J, Zhou H, Liao JY, Ma LM, Chen YQ, Qu LH 2008. Stressinduced tRNA-derived RNAs: a novel class of small RNAs in the primitive eukaryote Giardia lamblia. Nucleic Acids Res 36: 6048-6055.

Michaeli S, Doniger T, Gupta SK, Wurtzel O, Romano M, Visnovezky D, Sorek R, Unger R, Ullu E 2012. RNA-seq analysis of small RNPs in Trypanosoma brucei reveals a rich repertoire of noncoding RNAs. Nucleic Acids Res 40: 1282-1298.

Minning TA, Weatherly DB, Atwood J 3rd, Orlando R, Tarleton RL 2009. The steady-state transcriptome of the four major life-cycle stages of Trypanosoma cruzi. BMC Genomics 10: 370.

Nardelli SC, Avila AR, Freund A, Motta MC, Manhaes L, de Jesus
TC, Schenkman S, Fragoso SP, Krieger MA, Goldenberg S, Dallagiovanna B 2007. Small-subunit rRNA processome proteins are translationally regulated during differentiation of Trypanosoma cruzi. Eukaryot Cell 6: 337-345.

Padilla-Mejia NE, Florencio-Martinez LE, Figueroa-Angulo EE, Manning-Cela RG, Hernandez-Rivas R, Myler PJ, Martinez-Calvillo S 2009. Gene organization and sequence analyses of transfer RNA genes in trypanosomatid parasites. BMC Genomics 10: 232.

Pederson T 2010. Regulatory RNAs derived from transfer RNA? RNA 16: $1865-1869$

Pucadyil TJ, Schmid SL 2009. Conserved functions of membrane active GTPases in coated vesicle formation. Science 325: 1217-1220.

Will S, Reiche K, Hofacker IL, Stadler PF, Backofen R 2007. Inferring noncoding RNA families and classes by means of genomescale structure-based clustering. PLoS Comput Biol 3: 680-691. 


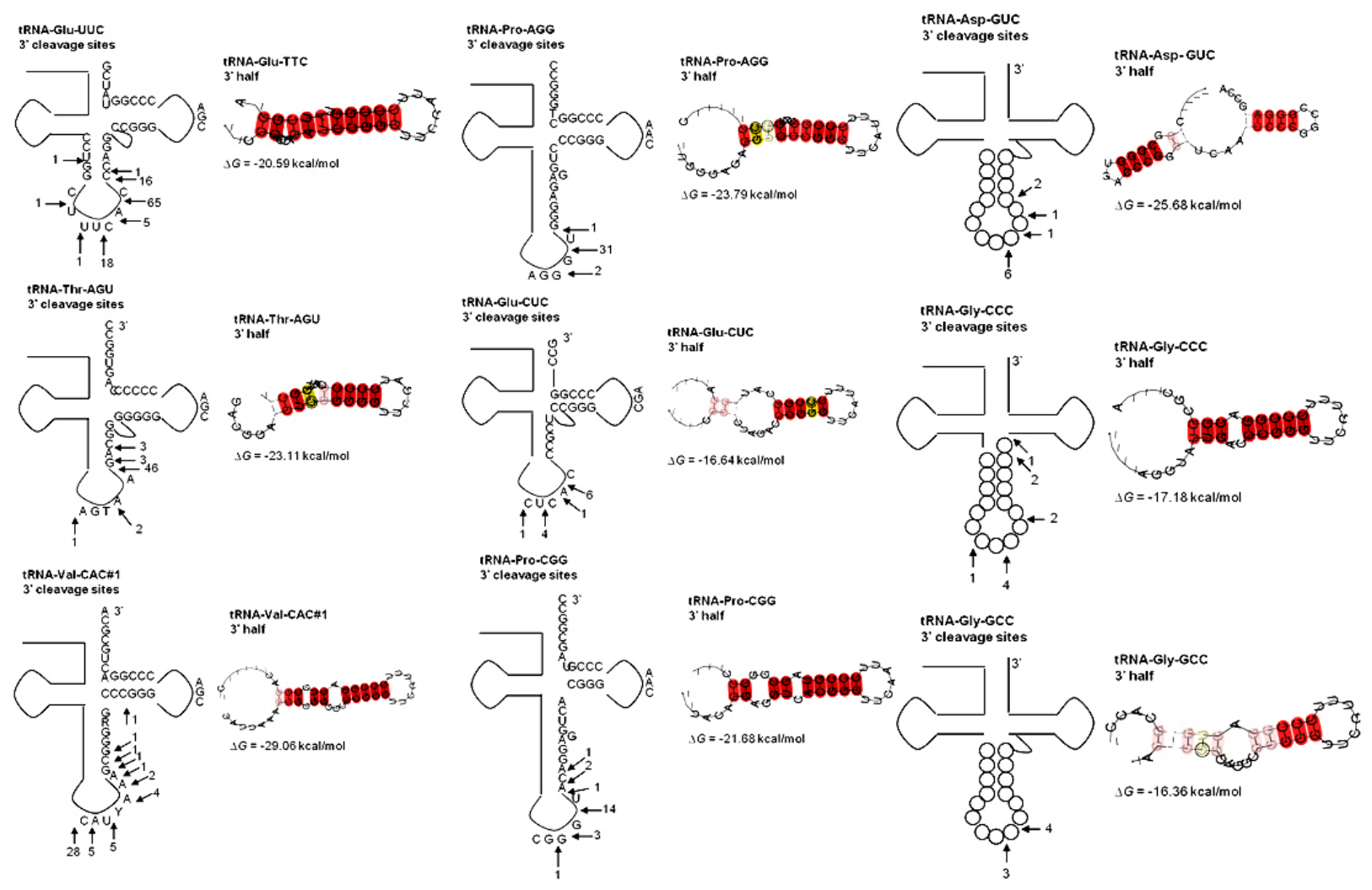

Predicted secondary structures adopted by the most abundant 3' tRNA fragments. 
TABLE

List of cloned tRNA-derived fragments, their identity obtained by Basic Local Alignment Search Tool against database sequences from Trypanosoma cruzi and if the sequence matches either the 5', mid portion or 3' portion of the tRNA

\begin{tabular}{|c|c|c|}
\hline Clone & tRNA & Cloned sequence \\
\hline 332 & tRNA-Glu:anticodon TTC, 3 ' & ACCAGGAGATCCGGGTTCGATTCCCGGTATCGGA \\
\hline 634 & tRNA-Glu:anticodon TTC, 3 ' & ACCCAGGAGATCCGGGTTCGATTCCCGGTATCGGA \\
\hline 70 & tRNA-Glu:anticodon TTC, 3 ' & ACCCAGGAGATCCGGGTTCGATTCCCGGTATCGGAACC \\
\hline 713 & tRNA-Glu:anticodon TTC, 3 ' & ACCCAGGAGATCCGGGTTCGATTCCCGGTATCGGAACC \\
\hline 831 & tRNA-Glu:anticodon TTC, 3 ' & ACCCAGGAGATCCGGGTTCGATTCCCGGTATCGGAACCA \\
\hline 247 & tRNA-Glu:anticodon TTC, 3' & ACCCAGGAGATCCGGGTTCGATTCCCGGTATCGGAACCA \\
\hline 172 & tRNA-Glu:anticodon TTC, 3 ' & CACCCAGGAGATCCGGGTTC \\
\hline 102 & tRNA-Glu:anticodon TTC, 3' & CACCCAGGAGATCCGGGTTC \\
\hline 803 & tRNA-Glu:anticodon TTC, 3' & CACCCAGGAGATCCGGGTTCGATTCCCGGTATCG \\
\hline 685 & tRNA-Glu:anticodon TTC, 3' & CACCCAGGAGATCCGGGTTCGATTCCCGGTATCGCA \\
\hline 133 & tRNA-Glu:anticodon TTC, 3 ' & CACCCAGGAGATCCGGGTTCGATTCCCGGTATCGGA \\
\hline 150 & tRNA-Glu:anticodon TTC, 3' & CACCCAGGAGATCCGGGTTCGATTCCCGGTATCGGA \\
\hline 513 & tRNA-Glu:anticodon TTC, 3 ' & CACCCAGGAGATCCGGGTTCGATTCCCGGTATCGGA \\
\hline 334 & tRNA-Glu:anticodon TTC, 3' & CACCCAGGAGATCCGGGTTCGATTCCCGGTATCGGAA \\
\hline 614 & tRNA-Glu:anticodon TTC, 3 ' & CACCCAGGAGATCCGGGTTCGATTCCCGGTATCGGAACC \\
\hline 637 & tRNA-Glu:anticodon TTC, 3' & CACCCAGGAGATCCGGGTTCGATTCCCGGTATCGGAACC \\
\hline 686 & tRNA-Glu:anticodon TTC, 3 ' & CACCCAGGAGATCCGGGTTCGATTCCCGGTATCGGAACCA \\
\hline 5 & tRNA-Glu:anticodon TTC, 3' & CACCCAGGAGATCCGGGTTCGATTCCCGGTATCGGAACCA \\
\hline 703 & tRNA-Glu:anticodon TTC, 3 ' & CACCCAGGAGATCCGGGTTGGATTCCCGGTATCGGA \\
\hline 387 & tRNA-Glu:anticodon TTC, 3' & CACCCAGGAGATCCGGGTTTGATTCCCGGTATCGGA \\
\hline 444 & tRNA-Glu:anticodon TTC, 3 ' & CAGGAGATCCGGGTTCGATTCCCGGTATCGGA \\
\hline 124 & tRNA-Glu:anticodon TTC, 3 ' & CCAGGAAATCCGGGTTCGATTCCCGGTATCGGAACC \\
\hline 487 & tRNA-Glu:anticodon TTC, 3 ' & CCAGGAGATCCGGGTTCGATTCCCGG \\
\hline 397 & tRNA-Glu:anticodon TTC, 3' & CCAGGAGATCCGGGTTCGATTCCCGGTATCG \\
\hline 352 & tRNA-Glu:anticodon TTC, 3' & CCAGGAGATCCGGGTTCGATTCCCGGTATCGG \\
\hline 456 & tRNA-Glu:anticodon TTC, 3 ' & CCAGGAGATCCGGGTTCGATTCCCGGTATCGG \\
\hline 481 & tRNA-Glu:anticodon TTC, 3' & CCAGGAGATCCGGGTTCGATTCCCGGTATCGG \\
\hline 516 & tRNA-Glu:anticodon TTC, 3' & CCAGGAGATCCGGGTTCGATTCCCGGTATCGG \\
\hline 319 & tRNA-Glu:anticodon TTC, 3' & CCAGGAGATCCGGGTTCGATTCCCGGTATCGGA \\
\hline 380 & tRNA-Glu:anticodon TTC, 3 ' & CCAGGAGATCCGGGTTCGATTCCCGGTATCGGA \\
\hline 399 & tRNA-Glu:anticodon TTC, 3' & CCAGGAGATCCGGGTTCGATTCCCGGTATCGGA \\
\hline 791 & tRNA-Glu:anticodon TTC, 3 ' & CCAGGAGATCCGGGTTCGATTCCCGGTATCGGA \\
\hline 792 & tRNA-Glu:anticodon TTC, 3' & CCAGGAGATCCGGGTTCGATTCCCGGTATCGGA \\
\hline 833 & tRNA-Glu:anticodon TTC, 3 ' & CCAGGAGATCCGGGTTCGATTCCCGGTATCGGAACCA \\
\hline 549 & tRNA-Glu:anticodon TTC, 3' & CCAGGAGATCCGGGTTCGATTCCCGGTATCGGAACCA \\
\hline 311 & tRNA-Glu:anticodon TTC, 3' & CCAGGAGATCCGGGTTCGATTCCCGGTATCGGAT \\
\hline 722 & tRNA-Glu:anticodon TTC, 3' & CCAGGAGATCCGGGTTGGATTCCCGGTATCGGAACCA \\
\hline 702 & tRNA-Glu:anticodon TTC, 3' & CCCAGGAGATCCGGGTTCGATTCCCGGGATAGGA \\
\hline 472 & tRNA-Glu:anticodon TTC, 3' & CCCAGGAGATCCGGGTTCGATTCCCGGGATCGG \\
\hline 427 & tRNA-Glu:anticodon TTC, 3' & CCCAGGAGATCCGGGTTCGATTCCCGGTATCG \\
\hline 87 & tRNA-Glu:anticodon TTC, 3' & CCCAGGAGATCCGGGTTCGATTCCCGGTATCGG \\
\hline 248 & tRNA-Glu:anticodon TTC, 3' & CCCAGGAGATCCGGGTTCGATTCCCGGTATCGG \\
\hline 331 & tRNA-Glu:anticodon TTC, 3 ' & CCCAGGAGATCCGGGTTCGATTCCCGGTATCGG \\
\hline 390 & tRNA-Glu:anticodon TTC, 3' & CCCAGGAGATCCGGGTTCGATTCCCGGTATCGG \\
\hline 429 & tRNA-Glu:anticodon TTC, 3' & CCCAGGAGATCCGGGTTCGATTCCCGGTATCGG \\
\hline 506 & tRNA-Glu:anticodon TTC, 3' & CCCAGGAGATCCGGGTTCGATTCCCGGTATCGG \\
\hline 602 & tRNA-Glu:anticodon TTC, 3' & CCCAGGAGATCCGGGTTCGATTCCCGGTATCGG \\
\hline 779 & tRNA-Glu:anticodon TTC, 3' & CCCAGGAGATCCGGGTTCGATTCCCGGTATCGG \\
\hline 793 & tRNA-Glu:anticodon TTC, 3' & CCCAGGAGATCCGGGTTCGATTCCCGGTATCGG \\
\hline 542 & tRNA-Glu:anticodon TTC, 3 ' & CCCAGGAGATCCGGGTTCGATTCCCGGTATCGG \\
\hline 117 & tRNA-Glu:anticodon TTC, 3' & CCCAGGAGATCCGGGTTCGATTCCCGGTATCGGA \\
\hline 151 & tRNA-Glu:anticodon TTC, 3' & CCCAGGAGATCCGGGTTCGATTCCCGGTATCGGA \\
\hline
\end{tabular}




\begin{tabular}{|c|c|c|}
\hline Clone & tRNA & Cloned sequence \\
\hline 164 & tRNA-Glu:anticodon TTC, 3' & CCCAGGAGATCCGGGTTCGATTCCCGGTATCGGA \\
\hline 252 & tRNA-Glu:anticodon TTC, 3' & CCCAGGAGATCCGGGTTCGATTCCCGGTATCGGA \\
\hline 354 & tRNA-Glu:anticodon TTC, 3' & CCCAGGAGATCCGGGTTCGATTCCCGGTATCGGA \\
\hline 402 & tRNA-Glu:anticodon TTC, 3' & CCCAGGAGATCCGGGTTCGATTCCCGGTATCGGA \\
\hline 403 & tRNA-Glu:anticodon TTC, 3' & CCCAGGAGATCCGGGTTCGATTCCCGGTATCGGA \\
\hline 407 & tRNA-Glu:anticodon TTC, 3' & CCCAGGAGATCCGGGTTCGATTCCCGGTATCGGA \\
\hline 408 & tRNA-Glu:anticodon TTC, 3' & CCCAGGAGATCCGGGTTCGATTCCCGGTATCGGA \\
\hline 416 & tRNA-Glu:anticodon TTC, 3' & CCCAGGAGATCCGGGTTCGATTCCCGGTATCGGA \\
\hline 419 & tRNA-Glu:anticodon TTC, 3' & CCCAGGAGATCCGGGTTCGATTCCCGGTATCGGA \\
\hline 424 & tRNA-Glu:anticodon TTC, 3' & CCCAGGAGATCCGGGTTCGATTCCCGGTATCGGA \\
\hline 439 & tRNA-Glu:anticodon TTC, 3' & CCCAGGAGATCCGGGTTCGATTCCCGGTATCGGA \\
\hline 452 & tRNA-Glu:anticodon TTC, 3' & CCCAGGAGATCCGGGTTCGATTCCCGGTATCGGA \\
\hline 466 & tRNA-Glu:anticodon TTC, 3' & CCCAGGAGATCCGGGTTCGATTCCCGGTATCGGA \\
\hline 480 & tRNA-Glu:anticodon TTC, 3' & CCCAGGAGATCCGGGTTCGATTCCCGGTATCGGA \\
\hline 483 & tRNA-Glu:anticodon TTC, 3' & CCCAGGAGATCCGGGTTCGATTCCCGGTATCGGA \\
\hline 485 & tRNA-Glu:anticodon TTC, 3' & CCCAGGAGATCCGGGTTCGATTCCCGGTATCGGA \\
\hline 492 & tRNA-Glu:anticodon TTC, 3' & CCCAGGAGATCCGGGTTCGATTCCCGGTATCGGA \\
\hline 494 & tRNA-Glu:anticodon TTC, 3' & CCCAGGAGATCCGGGTTCGATTCCCGGTATCGGA \\
\hline 505 & tRNA-Glu:anticodon TTC, 3' & CCCAGGAGATCCGGGTTCGATTCCCGGTATCGGA \\
\hline 507 & tRNA-Glu:anticodon TTC, 3' & CCCAGGAGATCCGGGTTCGATTCCCGGTATCGGA \\
\hline 511 & tRNA-Glu:anticodon TTC, 3' & CCCAGGAGATCCGGGTTCGATTCCCGGTATCGGA \\
\hline 515 & tRNA-Glu:anticodon TTC, 3' & CCCAGGAGATCCGGGTTCGATTCCCGGTATCGGA \\
\hline 519 & tRNA-Glu:anticodon TTC, 3' & CCCAGGAGATCCGGGTTCGATTCCCGGTATCGGA \\
\hline 701 & tRNA-Glu:anticodon TTC, 3' & CCCAGGAGATCCGGGTTCGATTCCCGGTATCGGA \\
\hline 727 & tRNA-Glu:anticodon TTC, 3' & CCCAGGAGATCCGGGTTCGATTCCCGGTATCGGA \\
\hline 734 & tRNA-Glu:anticodon TTC, 3' & CCCAGGAGATCCGGGTTCGATTCCCGGTATCGGA \\
\hline 749 & tRNA-Glu:anticodon TTC, 3' & CCCAGGAGATCCGGGTTCGATTCCCGGTATCGGA \\
\hline 751 & tRNA-Glu:anticodon TTC, 3' & CCCAGGAGATCCGGGTTCGATTCCCGGTATCGGA \\
\hline 776 & tRNA-Glu:anticodon TTC, 3' & CCCAGGAGATCCGGGTTCGATTCCCGGTATCGGA \\
\hline 790 & tRNA-Glu:anticodon TTC, 3' & CCCAGGAGATCCGGGTTCGATTCCCGGTATCGGA \\
\hline 834 & tRNA-Glu:anticodon TTC, 3' & CCCAGGAGATCCGGGTTCGATTCCCGGTATCGGA \\
\hline 840 & tRNA-Glu:anticodon TTC, 3' & CCCAGGAGATCCGGGTTCGATTCCCGGTATCGGA \\
\hline 141 & tRNA-Glu:anticodon TTC, 3' & CCCAGGAGATCCGGGTTCGATTCCCGGTATCGGA \\
\hline 25 & tRNA-Glu:anticodon TTC, 3' & CCCAGGAGATCCGGGTTCGATTCCCGGTATCGGA \\
\hline 357 & tRNA-Glu:anticodon TTC, 3' & CCCAGGAGATCCGGGTTCGATTCCCGGTATCGGAA \\
\hline 7 & tRNA-Glu:anticodon TTC, 3' & CCCAGGAGATCCGGGTTCGATTCCCGGTATCGGAAC \\
\hline 652 & tRNA-Glu:anticodon TTC, 3' & CCCAGGAGATCCGGGTTCGATTCCCGGTATCGGAAC \\
\hline 710 & tRNA-Glu:anticodon TTC, 3' & CCCAGGAGATCCGGGTTCGATTCCCGGTATCGGAACC \\
\hline 626 & tRNA-Glu:anticodon TTC, 3' & CCCAGGAGATCCGGGTTCGATTCCCGGTATCGGAACC \\
\hline 656 & tRNA-Glu:anticodon TTC, 3' & CCCAGGAGATCCGGGTTCGATTCCCGGTATCGGAACC \\
\hline 342 & tRNA-Glu:anticodon TTC, 3' & CCCAGGAGATCCGGGTTCGATTCCCGGTATCGGAACC \\
\hline 647 & tRNA-Glu:anticodon TTC, 3' & CCCAGGAGATCCGGGTTCGATTCCCGGTATCGGAACC \\
\hline 9 & tRNA-Glu:anticodon TTC, 3' & CCCAGGAGATCCGGGTTCGATTCCCGGTATCGGAACC \\
\hline 479 & tRNA-Glu:anticodon TTC, 3' & CCCAGGAGATCCGGGTTTGATTCCCGGTATCGG \\
\hline 484 & tRNA-Glu:anticodon TTC, 3' & CCCGCTAGACCCGGATTCATTTCCCGGCATCGGA \\
\hline 774 & tRNA-Glu:anticodon TTC, 3' & CCCTCGAGATCCGGGTTCGATTCCCCGTCTCGGA \\
\hline 375 & tRNA-Glu:anticodon TTC, 3’ & CTCAGGAGATCCGGGTTCGATTCCCAA \\
\hline 283 & tRNA-Glu:anticodon TTC, 3' & TCCAGGAGATCCGGGTTCGATTCCCGGTATC \\
\hline 760 & tRNA-Glu:anticodon TTC, 3' & TCCGATATGGTATAACGGTTAGAACACCTGGC \\
\hline 708 & tRNA-Glu:anticodon TTC, 3' & TCCGATATGGTATAACGGTTAGAACACCTGGC \\
\hline 442 & tRNA-Glu:anticodon TTC, 3' & TCCGATATGGTATAACGGTTAGAACACCTGGC \\
\hline 348 & tRNA-Glu:anticodon TTC, 3' & TCCGATATGGTATAACGGTTGGAGCCAACAGGC \\
\hline 184 & tRNA-Glu:anticodon TTC, 3' & CACCCAGGAGATCCGGGTTCGATTCCCGGTATCGGAA \\
\hline 314 & tRNA-Glu:anticodon TTC, 3' & TTCACCCAGGAGATCCGGGTTCGATTCCCGGTATCGGAAC \\
\hline
\end{tabular}




\begin{tabular}{|c|c|c|}
\hline Clone & tRNA & Cloned sequence \\
\hline 339 & tRNA-Glu:anticodon TTC, 5 , & CACCCAGGAGATCCGGGTTCGATTCCCGGTATCGGAACC \\
\hline 723 & tRNA-Glu:anticodon TTC, 5 & CCGATATGGTATAACGGTTAGAACACCCGGC \\
\hline 860 & tRNA-Glu:anticodon TTC, 5 & CCGATATGGTATAACGGTTAGAACACCTGG \\
\hline 778 & tRNA-Glu:anticodon TTC, 5' & CCGATATGGTATAACGGTTAGAACACCTGGC \\
\hline 282 & tRNA-Glu:anticodon TTC, 5 & CCGATATGGTATAACGGTTAGAACACCTGGC \\
\hline 520 & tRNA-Glu:anticodon TTC, 5 & CCGATATGGTATAACGGTTAGAACACCTGGC \\
\hline 580 & tRNA-Glu:anticodon TTC, 5 & CGATATGGTATAACGGTTAGAACACCTGG \\
\hline 471 & tRNA-Glu:anticodon TTC, 5 & TCCGATATGGTATAACGGTT \\
\hline 623 & tRNA-Glu:anticodon TTC, 5 & TCCGATATGGTATAACGGTTAGAACACCTGGC \\
\hline 358 & tRNA-Glu:anticodon TTC, 5 & TCCGATATGGTATAACGGTTAGAACACCTGGC \\
\hline 425 & tRNA-Glu:anticodon TTC, 5 & TCCGATATGGTATAACGGTTAGAACACCTGGC \\
\hline 495 & tRNA-Glu:anticodon TTC, 5 & TCCGATATGGTATAACGGTTAGAACACCTGGC \\
\hline 568 & tRNA-Glu:anticodon TTC, 5' & TCCGATATGGTATAACGGTTAGAACACCTGGC \\
\hline 225 & tRNA-Thr:anticodon AGT, 3' & AAAGGAGGTCGGGGGTTCGATCCCCCCAGTGGCC \\
\hline 601 & tRNA-Thr:anticodon AGT, 3' & AAGACGGAGGTCGGGGGTTCGATCCCCCCAGTGGC \\
\hline 817 & tRNA-Thr:anticodon AGT, 3' & AAGACGGAGGTCGGGGGTTCGATCCCCCCAGTGGCCTCCA \\
\hline 355 & tRNA-Thr:anticodon AGT, 3' & ACGGAGGTCGGGGGTTCGATCCCCCCAGTGGCC \\
\hline 512 & tRNA-Thr:anticodon AGT, 3' & ACGGAGGTCGGGGGTTCGATCCCCCCAGTGGCC \\
\hline 251 & tRNA-Thr:anticodon AGT, 3' & CGGAGGTCGGGGGTTCGATCCCCCCAGTGGCC \\
\hline 398 & tRNA-Thr:anticodon AGT, 3' & CGGAGGTCGGGGGTTCGATCCCCCCAGTGGCC \\
\hline 720 & tRNA-Thr:anticodon AGT, 3' & CGGAGGTCGGGGGTTCGATCCCCCCAGTGGCC \\
\hline 795 & tRNA-Thr:anticodon AGT, 3' & GACGGAGGTCAGGGGTTCGATCCCCCCAGTGGCCT \\
\hline 781 & tRNA-Thr:anticodon AGT, 3' & GACGGAGGTCGGGGGTTAGATCCCCCCAGCGGCCT \\
\hline 567 & tRNA-Thr:anticodon AGT, 3' & GACGGAGGTCGGGGGTTCGATCCCCCCAGTGGC \\
\hline 595 & tRNA-Thr:anticodon AGT, 3' & GACGGAGGTCGGGGGTTCGATCCCCCCAGTGGC \\
\hline 78 & tRNA-Thr:anticodon AGT, 3' & GACGGAGGTCGGGGGTTCGATCCCCCCAGTGGCC \\
\hline 160 & tRNA-Thr:anticodon AGT, 3' & GACGGAGGTCGGGGGTTCGATCCCCCCAGTGGCC \\
\hline 185 & tRNA-Thr:anticodon AGT, 3' & GACGGAGGTCGGGGGTTCGATCCCCCCAGTGGCC \\
\hline 443 & tRNA-Thr:anticodon AGT, 3' & GACGGAGGTCGGGGGTTCGATCCCCCCAGTGGCC \\
\hline 474 & tRNA-Thr:anticodon AGT, 3' & GACGGAGGTCGGGGGTTCGATCCCCCCAGTGGCC \\
\hline 490 & tRNA-Thr:anticodon AGT, 3' & GACGGAGGTCGGGGGTTCGATCCCCCCAGTGGCC \\
\hline 498 & tRNA-Thr:anticodon AGT, 3' & GACGGAGGTCGGGGGTTCGATCCCCCCAGTGGCC \\
\hline 526 & tRNA-Thr:anticodon AGT, 3' & GACGGAGGTCGGGGGTTCGATCCCCCCAGTGGCC \\
\hline 546 & tRNA-Thr:anticodon AGT, 3' & GACGGAGGTCGGGGGTTCGATCCCCCCAGTGGCC \\
\hline 575 & tRNA-Thr:anticodon AGT, 3' & GACGGAGGTCGGGGGTTCGATCCCCCCAGTGGCC \\
\hline 635 & tRNA-Thr:anticodon AGT, 3' & GACGGAGGTCGGGGGTTCGATCCCCCCAGTGGCC \\
\hline 719 & tRNA-Thr:anticodon AGT, 3' & GACGGAGGTCGGGGGTTCGATCCCCCCAGTGGCC \\
\hline 728 & tRNA-Thr:anticodon AGT, 3' & GACGGAGGTCGGGGGTTCGATCCCCCCAGTGGCC \\
\hline 761 & tRNA-Thr:anticodon AGT, 3' & GACGGAGGTCGGGGGTTCGATCCCCCCAGTGGCC \\
\hline 846 & tRNA-Thr:anticodon AGT, 3' & GACGGAGGTCGGGGGTTCGATCCCCCCAGTGGCC \\
\hline 851 & tRNA-Thr:anticodon AGT, 3' & GACGGAGGTCGGGGGTTCGATCCCCCCAGTGGCC \\
\hline 13 & tRNA-Thr:anticodon AGT, 3' & GACGGAGGTCGGGGGTTCGATCCCCCCAGTGGCC \\
\hline 56 & tRNA-Thr:anticodon AGT, 3' & GACGGAGGTCGGGGGTTCGATCCCCCCAGTGGCC \\
\hline 63 & tRNA-Thr:anticodon AGT, 3' & GACGGAGGTCGGGGGTTCGATCCCCCCAGTGGCC \\
\hline 85 & tRNA-Thr:anticodon AGT, 3' & GACGGAGGTCGGGGGTTCGATCCCCCCAGTGGCC \\
\hline 551 & tRNA-Thr:anticodon AGT, 3' & GACGGAGGTCGGGGGTTCGATCCCCCCAGTGGCC \\
\hline 61 & tRNA-Thr:anticodon AGT, 3' & GACGGAGGTCGGGGGTTCGATCCCCCCAGTGGCCT \\
\hline 2 & tRNA-Thr:anticodon AGT, 3' & GACGGAGGTCGGGGGTTCGATCCCCCCAGTGGCCT \\
\hline 96 & tRNA-Thr:anticodon AGT, 3' & GACGGAGGTCGGGGGTTCGATCCCCCCAGTGGCCT \\
\hline 120 & tRNA-Thr:anticodon AGT, 3' & GACGGAGGTCGGGGGTTCGATCCCCCCAGTGGCCT \\
\hline 318 & tRNA-Thr:anticodon AGT, 3' & GACGGAGGTCGGGGGTTCGATCCCCCCAGTGGCCT \\
\hline 423 & tRNA-Thr:anticodon AGT, 3' & GACGGAGGTCGGGGGTTCGATCCCCCCAGTGGCCT \\
\hline 455 & tRNA-Thr:anticodon AGT, 3' & GACGGAGGTCGGGGGTTCGATCCCCCCAGTGGCCT \\
\hline 504 & tRNA-Thr:anticodon AGT, 3' & GACGGAGGTCGGGGGTTCGATCCCCCCAGTGGCCT \\
\hline
\end{tabular}




\begin{tabular}{|c|c|c|}
\hline Clone & tRNA & Cloned sequence \\
\hline 578 & tRNA-Thr:anticodon AGT, 3' & GACGGAGGTCGGGGGTTCGATCCCCCCAGTGGCCT \\
\hline 721 & tRNA-Thr:anticodon AGT, 3 ' & GACGGAGGTCGGGGGTTCGATCCCCCCAGTGGCCT \\
\hline 745 & tRNA-Thr:anticodon AGT, 3 ' & GACGGAGGTCGGGGGTTCGATCCCCCCAGTGGCCT \\
\hline 780 & tRNA-Thr:anticodon AGT, 3' & GACGGAGGTCGGGGGTTCGATCCCCCCAGTGGCCT \\
\hline 176 & tRNA-Thr:anticodon AGT, 3' & GACGGAGGTCGGGGGTTCGATCCCCCCAGTGGCCT \\
\hline 345 & tRNA-Thr:anticodon AGT, 3' & GACGGAGGTCGGGGGTTCGATCCCCCCAGTGGCCTC \\
\hline 295 & tRNA-Thr:anticodon AGT, 3 ' & GACGGAGGTCGGGGGTTCGATCCCCCCAGTGGCCTCCA \\
\hline 530 & tRNA-Thr:anticodon AGT, 3 ' & GACGGAGGTCGGGGGTTCGATCCCCCCAGTGGCCTCCA \\
\hline 785 & tRNA-Thr:anticodon AGT, 3 ' & GACGGAGGTCGGGGGTTCGATCCCCCCAGTGGCCTCCA \\
\hline 593 & tRNA-Thr:anticodon AGT, 3' & GACGGAGGTCGGGGGTTCGATCCCCCCTGCGGA \\
\hline 799 & tRNA-Thr:anticodon AGT, 3' & GACGGAGGTCGGGGTTCGATCCCCCCAGTGGCCT \\
\hline 818 & tRNA-Thr:anticodon AGT, 3' & GACGGAGGTTGGGGGTTCGATCCCCCCAGTGGCC \\
\hline 271 & tRNA-Val:anticodon CAC\#1, 3' & AAAGCGGGGGGCCCGGGTTCGTTTCCCGGACTGCGCAC \\
\hline 784 & tRNA-Val:anticodon CAC\#1, 3' & AAAGCGGGGGGCCCGGGTTCGTTTCCCGGACTGCGCAC \\
\hline 737 & tRNA-Val:anticodon CAC\#1, 3' & AAAGCGGGGGGCCCGGGTTCGTTTCCCGGACTGCGCACC \\
\hline 321 & tRNA-Val:anticodon CAC\#1, 3' & AAAGCGGGGGGCCCGGGTTCGTTTCCCGGACTGCGCACCA \\
\hline 577 & tRNA-Val:anticodon CAC\#1, 3' & AAGCGGGGGGCCCGGGTTCGTTTCCCGGACTGCGCAC \\
\hline 532 & tRNA-Val:anticodon CAC\#1, 3' & ACGCGGGAGGCCCGGGTTCGTTTCCCGGACTGCGCAC \\
\hline 246 & tRNA-Val:anticodon CAC\#1, 3' & CAAAGCGGGGGGCCCGGGTTCGATTCCCGGACTGCGCACC \\
\hline 197 & tRNA-Val:anticodon CAC\#1, 3' & CAAAGCGGGGGGCCCGGGTTCGTTTCCCGGACTGCGCAC \\
\hline 581 & tRNA-Val:anticodon CAC\#1, 3' & CAAAGCGGGGGGCCCGGGTTCGTTTCCCGGACTGCGCAC \\
\hline 137 & tRNA-Val:anticodon CAC\#1, 3' & CAAAGCGGGGGGCCCGGGTTCGTTTCCCGGACTGCGCACC \\
\hline 261 & tRNA-Val:anticodon CAC\#1, 3' & CAAAGCGGGGGGCCCGGGTTCGTTTCCCGGACTGCGCACC \\
\hline 564 & tRNA-Val:anticodon CAC\#1, 3' & CATCAAAGCGGGGGGCCCGGGTTTCCCGGACTGCGCACCA \\
\hline 356 & tRNA-Val:anticodon CAC\#1, 3' & CGCGGGAGGCCCGGGTTCGTTTCCCGGACTGCGCA \\
\hline 679 & tRNA-Val:anticodon CAC\#1, 3' & CGGGGGGCCCGGGTTTTTCCCGGACTGCGCAC \\
\hline 540 & tRNA-Val:anticodon CAC\#1, 3' & GCGGGGGGCCCGGGTTCGATTCCCGGACTGCGCAC \\
\hline 289 & tRNA-Val:anticodon CAC\#1, 3' & GGGCCCGGGTTCGTTTCCCGGACTGCGCACCAT \\
\hline 717 & tRNA-Val:anticodon CAC\#1, 3' & GGGGGGCCCGGGTTCGTTTCCCGGACTGCGCACC \\
\hline 450 & tRNA-Pro:anticodon AGG, 3 ' & GTGGGAGAGGTCCCGGGTTTCCCGGCTGGGCCC \\
\hline 861 & tRNA-Pro:anticodon AGG, 3' & TGGGAGAGGTCCCGGGTTTTTCCCGGCTGGGCC \\
\hline 393 & tRNA-Pro:anticodon AGG, 3 ' & ACAGGAGGTCACGGGTTCATTTCCCGGCTGGGCCCCC \\
\hline 335 & tRNA-Pro:anticodon AGG, 3' & GGGAGAGGTCCCGGGTTCATTTCCCCGCTGGCCCC \\
\hline 410 & tRNA-Pro:anticodon AGG, 3' & TGGGAGAGGTCCCGGCTTCAGTTCCCGGCTGGATCCC \\
\hline 92 & tRNA-Pro:anticodon AGG, 3 ' & TGGGAGAGGTCCCGGGTTCAATTCCCGGCTGGGCCC \\
\hline 180 & tRNA-Pro:anticodon AGG, 3 ' & TGGGAGAGGTCCCGGGTTCAATTCCCGGCTGGGCCC \\
\hline 527 & tRNA-Pro:anticodon AGG, 3' & TGGGAGAGGTCCCGGGTTCAATTCCCGGCTGGGCCC \\
\hline 250 & tRNA-Pro:anticodon AGG, 3' & TGGGAGAGGTCCCGGGTTCATTTCCCGGCTGGGCC \\
\hline 67 & tRNA-Pro:anticodon AGG, 3 ' & TGGGAGAGGTCCCGGGTTCATTTCCCGGCTGGGCCC \\
\hline 69 & tRNA-Pro:anticodon AGG, 3 ' & TGGGAGAGGTCCCGGGTTCATTTCCCGGCTGGGCCC \\
\hline 337 & tRNA-Pro:anticodon AGG, 3 ' & TGGGAGAGGTCCCGGGTTCATTTCCCGGCTGGGCCC \\
\hline 361 & tRNA-Pro:anticodon AGG, 3 ' & TGGGAGAGGTCCCGGGTTCATTTCCCGGCTGGGCCC \\
\hline 370 & tRNA-Pro:anticodon AGG, 3 ' & TGGGAGAGGTCCCGGGTTCATTTCCCGGCTGGGCCC \\
\hline 379 & tRNA-Pro:anticodon AGG, 3 ' & TGGGAGAGGTCCCGGGTTCATTTCCCGGCTGGGCCC \\
\hline 384 & tRNA-Pro:anticodon AGG, 3' & TGGGAGAGGTCCCGGGTTCATTTCCCGGCTGGGCCC \\
\hline 400 & tRNA-Pro:anticodon AGG, 3 ' & TGGGAGAGGTCCCGGGTTCATTTCCCGGCTGGGCCC \\
\hline 517 & tRNA-Pro:anticodon AGG, 3 ' & TGGGAGAGGTCCCGGGTTCATTTCCCGGCTGGGCCC \\
\hline 651 & tRNA-Pro:anticodon AGG, 3' & TGGGAGAGGTCCCGGGTTCATTTCCCGGCTGGGCCC \\
\hline 665 & tRNA-Pro:anticodon AGG, 3 ' & TGGGAGAGGTCCCGGGTTCATTTCCCGGCTGGGCCC \\
\hline 762 & tRNA-Pro:anticodon AGG, 3 ' & TGGGAGAGGTCCCGGGTTCATTTCCCGGCTGGGCCC \\
\hline 775 & tRNA-Pro:anticodon AGG, 3 ' & TGGGAGAGGTCCCGGGTTCATTTCCCGGCTGGGCCC \\
\hline 191 & tRNA-Pro:anticodon AGG, 3' & TGGGAGAGGTCCCGGGTTCATTTCCCGGCTGGGCCCC \\
\hline 349 & tRNA-Pro:anticodon AGG, 3 ' & TGGGAGAGGTCCCGGGTTCATTTCCCGGCTGGGCCCC \\
\hline 422 & tRNA-Pro:anticodon AGG, 3' & TGGGAGAGGTCCCGGGTTCATTTCCCGGCTGGGCCCC \\
\hline
\end{tabular}




\begin{tabular}{|c|c|c|}
\hline Clone & tRNA & Cloned sequence \\
\hline 431 & tRNA-Pro:anticodon AGG, 3 ' & TGGGAGAGGTCCCGGGTTCATTTCCCGGCTGGGCCCC \\
\hline 458 & tRNA-Pro:anticodon AGG, 3' & TGGGAGAGGTCCCGGGTTCATTTCCCGGCTGGGCCCC \\
\hline 664 & tRNA-Pro:anticodon AGG, 3' & TGGGAGAGGTCCCGGGTTCATTTCCCGGCTGGGCCCC \\
\hline 189 & tRNA-Pro:anticodon AGG, 3 ' & TGGGAGAGGTCCCGGGTTCATTTCCCGGCTGGGCCCC \\
\hline 272 & tRNA-Pro:anticodon AGG, 3 ' & TGGGAGAGGTCCCGGGTTCATTTCCCGGCTGGGCCCCC \\
\hline 814 & tRNA-Pro:anticodon AGG, 3 ' & TGGGAGAGGTCCCGGGTTCATTTCCCGGCTGGGCCCCC \\
\hline 824 & tRNA-Pro:anticodon AGG, 3 ' & TGGGAGAGGTCCCGGGTTCATTTCCCGGCTGGGCCCCC \\
\hline 396 & tRNA-Pro:anticodon AGG, 3' & TGGGGGAGGTCCCGGGTTCATTTCCCGGCGGGGCCC \\
\hline 496 & tRNA-Glu:anticodon CTC, 3 ' & ACCCGCTAGACCCGGGTTC.ATTTCCCGGCATCGGA \\
\hline 159 & tRNA-Glu:anticodon CTC, 3 ' & CACCCAGGAGATCCGGGTTCGATTCCCGGTATCGG \\
\hline 128 & tRNA-Glu:anticodon CTC, $3^{\prime}$ & CACCCGCTAGACCCGGGTTC.ATTTCCCGGCATCGGAA \\
\hline 669 & tRNA-Glu:anticodon CTC, 3 ' & CACCCGCTAGACCCGGGTTCATTTCCCGGCATCGGAACCA \\
\hline 765 & tRNA-Glu:anticodon CTC, 3 ' & CACCCGGTAGACCCGGGTTCATTTCCCGTCATCGGAACCA \\
\hline 338 & tRNA-Glu:anticodon CTC, 3 ' & CCCAGGAGATCCGGGTTCGATTCCCGGTATCGGA \\
\hline 378 & tRNA-Glu:anticodon CTC, 3 ' & CCCAGGAGATCCGGGTTCGATTCCCGGTATCGGA \\
\hline 453 & tRNA-Glu:anticodon CTC, 3' & CCCGCTAGACCCGGGTTCATTTCCCGGCATCGG \\
\hline 486 & tRNA-Glu:anticodon CTC, 3' & CCCGCTAGACCCGGGTTCATTTCCCGGCATCGGA \\
\hline 571 & tRNA-Glu:anticodon CTC, 3 ' & CCCGCTAGACCCGGGTTCATTTCTCGGCATC \\
\hline 820 & tRNA-Glu:anticodon CTC, 3 ' & CCCGCTAGACCCGGGTTCATTTCTCGGCATC \\
\hline 536 & tRNA-Glu:anticodon CTC, 5 & CCGGTGTGGTATAGTGGTTAGAACAAGCGGC \\
\hline 692 & tRNA-Glu:anticodon CTC, 5 & CCGGTGTGGTATAGTGGTTAGAACAAGCGG \\
\hline 598 & tRNA-Glu:anticodon CTC, 5 & CCGGTGTGGTATAGTGGTTAGAACAAGCGGC \\
\hline 340 & tRNA-Glu:anticodon CTC, 5 & TCCGGTGTGGTATAGTGGTTAGAACAAGCGGC \\
\hline 543 & tRNA-Glu:anticodon CTC, 5 & TCCGGTGTGGTATAGTGGCTAGAACAAGCGGC \\
\hline 259 & tRNA-Glu:anticodon CTC, 5 & TCCGGTGTGGTATAGTGGCTAGAACAAGCGGC \\
\hline 426 & tRNA-Glu:anticodon CTC, 5 & TCCGGTGTGGTATAGTGGCTAGAACAAGCGGC \\
\hline 454 & tRNA-Glu:anticodon CTC, 5 & TCCGGTGTGGTATAGTGGCTAGAACAAGCGGC \\
\hline 508 & tRNA-Glu:anticodon CTC, 5 & TCCGGTGTGGTATAGTGGCTAGAACAAGCGGCTC \\
\hline 343 & tRNA-Glu:anticodon CTC, 5 & TCCGGTGTGGTATAGTGGTTAGAACAAGCGGC \\
\hline 437 & tRNA-Glu:anticodon CTC, 5 & TCCGGTGTGGTATAGTGGTTAGAACAAGCGGC \\
\hline 459 & tRNA-Glu:anticodon CTC, mid & TAGTGGTTAGAACAAGCGGC \\
\hline 666 & tRNA-Asp:anticodon GTC, 3' & ACGCGGGTGACCCGGGTTCAATTCCCGGCCGGGAAGCCA \\
\hline 239 & tRNA-Asp:anticodon GTC, 3 ' & CACGCGGGAGACCCGGGTTCCTTT \\
\hline 612 & tRNA-Asp:anticodon GTC, 3' & CACGCGGGTGACCCGGGTTCAATTCCCGGCCGGGA \\
\hline 752 & tRNA-Asp:anticodon GTC, 3 ' & CACGCGGGTGACCCGGGTTCAATTCCCGGCCGGGAAGCCA \\
\hline 77 & tRNA-Asp:anticodon GTC, 3 ' & CACGCGGGTGACCCGGGTTCATTTCCCGGCCGGGAAGCCA \\
\hline 131 & tRNA-Asp:anticodon GTC, 3 ' & CGCGGGTGACCCGGGTTCAATTCCCGGCCGGGAAGCC \\
\hline 430 & tRNA-Asp:anticodon GTC, 3' & GCGGGTGACCCGGGTTCAATTCCCGGCCGGGA \\
\hline 447 & tRNA-Asp:anticodon GTC, 3 ' & GCGGGTGACCCGGGTTCAATTCCCGGCCGGGA \\
\hline 155 & tRNA-Asp:anticodon GTC, 3 ' & CACGCGGGTGACCCGGGTTCAATTCCCGGCCGGGAAGCCA \\
\hline 156 & tRNA-Asp:anticodon GTC, 3' & CACGCGGGTGACCCGGGTTCAATTCCCGGCCGGGAAGCCA \\
\hline 620 & tRNA-Asp:anticodon GTC, 5 & CTCGGTAGTATAATGGTAAGTATACCCGCC \\
\hline 4 & tRNA-Asp:anticodon GTC, 5 & CTCGGTAGTATAGTGGTAAGTATACCCGCC \\
\hline 57 & tRNA-Asp:anticodon GTC, 5 & CTCGGTAGTATAGTGGTAAGTATACCCGCC \\
\hline 500 & tRNA-Asp:anticodon GTC, 5' & CTCGGTAGTATAGTGGTAAGTATACCCGCC \\
\hline 46 & tRNA-Asp:anticodon GTC, 5 & TCTCGGTAGTATAGTGGTAAGTATACCCGCC \\
\hline 254 & tRNA-Asp:anticodon GTC, 5 , & TCGGTAGTATAGTGGTAAGTATACCCGCC \\
\hline 320 & tRNA-Asp:anticodon GTC, 5 & TCGGTAGTATAGTGGTAAGTATACCCGCC \\
\hline 88 & tRNA-Asp:anticodon GTC, 5 & TCTCGGTAGTATAGTGGTAAGTATACCCGC \\
\hline 678 & tRNA-Asp:anticodon GTC, 5 & TCTCGGTAGTATAGTGGTAAGTATACCCGC \\
\hline 363 & tRNA-Asp:anticodon GTC, 5 & TCTCGGTAGTATAGTGGTAAGTATACCCGCCT \\
\hline 741 & tRNA-Pro:anticodon CGG, 3' & AGGAGGTCACGGGTTCAATTCCCGTAGCGGCC \\
\hline 368 & tRNA-Pro:anticodon CGG, 3' & CAGGAGGTCACGGGTTCAATTCCCGTAGCGGCCC \\
\hline 470 & tRNA-Pro:anticodon CGG, 3 ' & CAGGAGGTCACGGGTTCATTTCCCGTAGCGGCCC \\
\hline
\end{tabular}




\begin{tabular}{|c|c|c|}
\hline Clone & tRNA & Cloned sequence \\
\hline 697 & tRNA-Pro:anticodon CGG, 3 ' & TACAGGAGGTCACGGGTTCATTTCCCGTAGCGGC \\
\hline 140 & tRNA-Pro:anticodon CGG, 3 ' & TACAGGAGGTCACGGGTTCAATTCCCGTAGCGGCC \\
\hline 71 & tRNA-Pro:anticodon CGG, 3 ' & TACAGGAGGTCACGGGTTCAATTCCCGTAGCGGCCC \\
\hline 351 & tRNA-Pro:anticodon CGG, 3 ' & TACAGGAGGTCACGGGTTCAATTCCCGTAGCGGCCC \\
\hline 636 & tRNA-Pro:anticodon CGG, 3 ' & TACAGGAGGTCACGGGTTCAATTCCCGTAGCGGCCC \\
\hline 350 & tRNA-Pro:anticodon CGG, 3 ' & TACAGGAGGTCACGGGTTCATTTCCCGTAGCGGCCC \\
\hline 420 & tRNA-Pro:anticodon CGG, 3 & TACAGGAGGTCACGGGTTCATTTCCCGTAGCGGCCC \\
\hline 457 & tRNA-Pro:anticodon CGG, 3 & TACAGGAGGTCACGGGTTCATTTCCCGTAGCGGCCC \\
\hline 465 & tRNA-Pro:anticodon CGG, 3 ' & GTACAGGAGGTCACGGGTTCAATTCCCGTAGCGGCCC \\
\hline 521 & tRNA-Pro:anticodon CGG, 3 & GTACAGGAGGTCACGGGTTCAATTCCCGTAGCGGCCC \\
\hline 84 & tRNA-Pro:anticodon CGG, 3 ' & TACAGGAGGTCACGGGTTCAATTCCCGTAGCGGCCCC \\
\hline 256 & tRNA-Pro:anticodon CGG, $3^{\prime}$ & TACAGGAGGTCACGGGTTCATTTCCCGTAGCGGCCCC \\
\hline 385 & tRNA-Pro:anticodon CGG, 3 ' & ACAGGAGGTCACGGGTTCATTTCCCGTAGCGGCCCCC \\
\hline 523 & tRNA-Pro:anticodon CGG, 3 & GGTACAGGAGGTCACGGGTTCAATTCCCGTAGCGGCCC \\
\hline 497 & tRNA-Pro:anticodon CGG, 3 ' & GTACAGGAGGTCACGGGTTCAATTCCCGTAGCGGCCCC \\
\hline 369 & tRNA-Pro:anticodon CGG, 3 ' & TACAGGAGGTCACGGGTTCAATTCCCGTAGCGGCCCCC \\
\hline 802 & tRNA-Pro:anticodon CGG, 3 ' & TACAGGAGGTCACGGGTTCATTTCCCGTAGCGGCCCCC \\
\hline 449 & tRNA-Pro:anticodon CGG, 3 ' & TACAGGAGGTCACGGGTTCATTTCCCGTAGCGGCCCCCA \\
\hline 460 & tRNA-Pro:anticodon CGG, 3' & TACAGGAGGTCACGGGTTCATTTCCCGTAGCGGCCCT \\
\hline 341 & tRNA-Pro:anticodon TGG, 3 ' & TACAGGAGGTCACGGGTTCAATTCCCGTAGCGGCCCA \\
\hline 138 & tRNA-Gly:anticodon CCC, 3 & AGGTATTGACCCGGGCTCGTTTCCCGGACGGCGCACCA \\
\hline 152 & tRNA-Gly:anticodon CCC, 3 ' & AGGTATTGACCCGGGTTCGTTTCCCGGACGGCGCA \\
\hline 16 & tRNA-Gly:anticodon CCC, 3' & CAAGGTATTGACCCGGGTTCGATTCCCGGACGGCGCA \\
\hline 34 & tRNA-Gly:anticodon CCC, 3 ' & CAAGGTATTGACCCGGGTTCGATTCCCGGACGGCGCA \\
\hline 590 & tRNA-Gly:anticodon CCC, 3 ' & CAAGGTATTGACCCGGGTTCGATTCCCGGACGGCGCA \\
\hline 114 & tRNA-Gly:anticodon CCC, 3 ' & CAAGGTATTGACCCGGGTTCGTTTCCCGGACGGCGCACCA \\
\hline 223 & tRNA-Gly:anticodon CCC, 3 ' & CCCAAGGTATTGACCCGGGTTCGTTTCCCGGACGGCGCA \\
\hline 473 & tRNA-Gly:anticodon CCC, 3 & TGACCCGGGTTCGTTTCCCGGACGGCGC \\
\hline 40 & tRNA-Gly:anticodon CCC, 3 ' & TTGACCCGGGTTCGTTTCCCGGACGGCGC \\
\hline 325 & tRNA-Gly:anticodon CCC, 3 & TTGACCCGGGTTCGTTTCCCGGACGGCGC \\
\hline 296 & tRNA-Gly:anticodon GCC, 3 ' & ACCCAGGAGATCCGGGTTCGATTCCCGGACTGCGCACC \\
\hline 233 & tRNA-Gly:anticodon GCC, 3' & ACGCGGGAGGCCCGGGTTCGTTTCCC \\
\hline 51 & tRNA-Gly:anticodon GCC, 3 & ACGCGGGAGGCCCGGGTTCGTTTCCCGGACTGCGCACC \\
\hline 857 & tRNA-Gly:anticodon GCC, 3 ' & CACGCGGGAGGCCCGGGTTCGATTCCCGGACTGCGCACCA \\
\hline 236 & tRNA-Gly:anticodon GCC, 3' & CACGCGGGAGGCCCGGGTTCGTTTCCCGGACTGCGCAC \\
\hline 74 & tRNA-Gly:anticodon GCC, 3' & CACGCGGGAGGCCCGGGTTCGTTTCCCGGACTGCGCACCA \\
\hline 53 & tRNA-Gly:anticodon GCC, mid & ACGAGGGAGGCCCGGGTT \\
\hline 798 & tRNA-His:anticodon GTG, 3 ' & CTCTGAATACCCGGGTTCGATTCCCGGTCTTCCCTCCA \\
\hline 418 & tRNA-His:anticodon GTG, 3 ' & CTGAATACCCGGATTCGATTCCCGGTCTTCCCTC \\
\hline 336 & tRNA-His:anticodon GTG, 3' & GTTCGATTCCCGGTCTTCCCT \\
\hline 260 & tRNA-Lys:anticodon TTT, 3' & AACCGTGTGGTCGTGGGTTCGATCCCCACGGATGGCAC \\
\hline 801 & tRNA-Ala:anticodon AGC, 3 ' & TGCGGGAGGTATTGGGATCGTTACCCAACTTCTCC \\
\hline 10 & tRNA-Ala:anticodon CGC, 3 ' & TACGGAAGGCCTAGGGTTCGATCCCCTACTCGTCCA \\
\hline 301 & tRNA-Ala:anticodon CGC, 3 ' & TACGGAAGGCCTAGGGTTCGATCCCCTACTCGTCCA \\
\hline 208 & tRNA-Val:anticodon CAC\#2, 5 , & GCGATGGTCGTCTAGGGGTTAGGACATTCTC \\
\hline 446 & tRNA-Val:anticodon CAC\#2, 5 , & GCGATGGTCGTCTAGTGGTTAGGACATTCGCC \\
\hline 856 & tRNA-Val:anticodon CAC\#2, 5 , & GCGATGGTCGTCTAGTGGTTAGGACATTCGC \\
\hline 395 & tRNA-Arg:anticodon TCG, 3 ' & CAGAGGGTTGCAGGTTCGGATCCTGTCACGGAT \\
\hline 381 & tRNA-Gly:anticodon TCC, 5 & GCAATCGTGGTCCAACGGTTAAGATCCCCGCC \\
\hline 428 & tRNA-Gly:anticodon TCC, 5 ' & GCAATCGTGGTCCAACGGTTAAGATCCCCGCC \\
\hline 573 & tRNA-Val:anticodon AAC 5, & GCGCTGATGGTCTAGGTGGTTATGACGTCGCTTTG \\
\hline 421 & tRNA-Val:anticodon AAC, 3 & GACACGGCGAAGGTCTCGGGTTCGGGTCCCGATCGGC \\
\hline 518 & tRNA-Trp:anticodon CCA, 3' & TCCAGGGGTCGCAGGTTCAATCCCTGCAGTCCTC \\
\hline 475 & tRNA-Tyr:anticodon GTA, 5 , & ССTTCTGTAGCTCAATTGGTAGAGCATGTGACTGTAG \\
\hline
\end{tabular}

the non-encoded nucleotides are in red. 